\title{
Inclusão do índice de espaçamento relativo no diagrama de manejo da densidade do povoamento
}

\author{
Inclusion of the relative spacing index in the stand density management \\ diagram
}

\section{Paulo Renato SchneiderI, Paulo Sérgio Pigato Schneider ${ }^{\mathrm{II}}$, Solon Jonas LonghiII, Junior Oliveira Mendes ${ }^{\text {IV }}$, Evandro Alcir Meyer ${ }^{\mathrm{V}}$}

\begin{abstract}
Resumo
Este estudo teve por objetivo estruturar a produção em volume de povoamentos de Pinus taeda L., através de diagrama de manejo, tomando como base os parâmetros: densidade populacional, altura dominante, diâmetro médio de área basal e índice de espaçamento relativo. Os dados utilizados são oriundos de 128 parcelas permanentes, as quais foram medidas anualmente até aos 18 anos de idade e conduzidas em densidade completa, com espaçamentos de 1,5 x 1,0 m e 1,5 x 2,0 m. Concomitantemente, foram utilizados dados de parcelas permanentes submetidas a diferentes intensidades de desbaste, sendo a densidade regulada pelo índice de espaçamento relativo. O diagrama de manejo elaborado permitiu a prognose do volume por hectare, do diâmetro médio de área basal e da disponibilidade de espaço de crescimento, através do índice de espaçamento relativo, a partir das variáveis altura dominante e número de árvores com precisão e baixo erro de estimativa.
\end{abstract}

Palavras-chave: Desbaste; Tolerância; Crescimento; Produção

\begin{abstract}
This study aimed to structure the production in volume of Pinus taeda L. stands, through a management diagram, based on the following parameters: population density, dominant height, average diameter of basal area and relative spacing index. The data used came from 128 permanent plots, which were measured annually up to 18 years of age and conducted at full density, with spacing of $1.5 \times 1.0 \mathrm{~m}$ and $1.5 \times 2.0 \mathrm{~m}$. At the same time, data from permanent plots subjected to different thinning intensities were used, and the density was regulated by the relative spacing index. The management diagram elaborated allowed the prognosis of the volume per hectare, the average diameter of the basal area and the availability of growth space, through the relative spacing index, from the variables dominant height and number of trees with precision and low error of estimate.
\end{abstract}

Keywords: Thinning; Tolerance; Growth; Yield

\footnotetext{
Engenheiro Florestal, Dr., Professor Titular do Departamento de Ciências Florestais, Centro de Ciências Rurais, Universidade Federal de Santa Maria, Av. Roraima, 1000, CEP 97105-900, Santa Maria (RS), Brasil. schneider.paulorenato@gmail.com (ORCID: 0000-0002-8955-3773)

Engenheiro Florestal, Dr., Bolsista de Pós-doutorado Junior do CNPq, Programa de Pós-graduação em Engenharia Florestal, Centro de Ciências Rurais, Universidade Federal de Santa Maria, Av. Roraima, 1000, CEP 97105-900, Santa Maria (RS), Brasil. paulosergiopigatoschneider@gmail.com (ORCID: 0000-0002-8317-5548)

III Engenheiro Florestal, Dr., Professor do Programa de Pós-graduação em Engenharia Florestal, Centro de Ciências Rurais, Universidade Federal de Santa Maria, Av. Roraima, 1000, CEP 97105-900, Santa Maria (RS), Brasil. longhi.solon@gmail.com (ORCID: 0000-0002-5701-2139)

Engenheiro Florestal, Dr., Programa de Pós-graduação em Engenharia Florestal, Centro de Ciências Rurais, Universidade Federal de Santa Maria, Av. Roraima, 1000, CEP 97105-900, Santa Maria (RS), Brasil. junioromendes@gmail.com (ORCID: 0000-0003-2744-6814)

Engenheiro Florestal, Dr., Programa de Pós-graduação em Engenharia Florestal, Centro de Ciências Rurais, Universidade Federal de Santa Maria, Av. Roraima, 1000, CEP 97105-900, Santa Maria (RS), Brasil. eam.meyer@gmail.com (ORCID: 0000-0003-4581-7690)
} 


\section{Introdução}

O controle populacional em povoamentos florestais normalmente é realizado pelo manejo da densidade que, a partir de um espaçamento inicial e posteriormente com a realização de desbastes, permite ao manejador ou empreendimento florestal alcançar os objetivos de produção estabelecidos. Esse processo exige que se conheçam os limites superior e inferior de estoque, sendo o primeiro utilizado para se obter um nível aceitável de crescimento das árvores e com vigor superior, enquanto que o último serve para manter um nível aceitável de ocupação do local.

Do ponto de vista biológico, os níveis de estoque de crescimento devem ser limitados dentro das densidades de suporte, que correspondem ao limiar de autodesbaste e fechamento do dossel (DEAN; BALDWIN, 1996). No entanto, traduzir os objetivos específicos de manejo para os níveis superior e inferior de estoque de crescimento é o passo mais complexo na determinação de um regime de manejo baseado na densidade (DAVIS, 1966).

Nesse sentido, buscando determinar a proporção máxima do número de plantas em relação à massa média, Yoda et al. (1963) formularam a lei de potência de $-3 / 2$, na qual a mortalidade dos indivíduos é dependente da densidade. A máxima densidade de plantas em relação à massa média, em plantas e em escala logarítmica, tem idêntica taxa de crescimento, para todos os locais, e o autodesbaste deve ocorrer quando as árvores tiverem uma completa ocupação do local. Esta lei da potência $-3 / 2$ é descrita por: $\ln (W)=\ln (C)-(3 / 2) \times \ln (N)$, em que: $\mathrm{N}=$ densidade, $\mathrm{W}=$ massa média e $\mathrm{C}=$ constante de proporcionalidade.

A teoria do autodesbaste foi estendida por Tang et al. (1994) para povoamentos conduzidos em densidade completa e subestocados. Segundo os autores, o autodesbaste inicia antes que seja atingida a linha de máxima densidade e a taxa de autodesbaste incrementa com a densidade. Essa lei de autodesbaste tem sido objeto de estudo para uso prático e de discussões acerca da sua eficiência no manejo florestal, por vários pesquisadores, como: Westoby (1981), Zeide (1987; 1991); Smith e Hann (1986); Del Rio et al. (2001), entre outros.

Embora os experimentos de campo sejam a melhor forma de determinar o momento da realização de desbastes e os limites teóricos mencionados acima, eles possuem duas sérias limitações: levam muitos anos para serem concluídos e os resultados não podem ser utilizados com precisão quando a qualidade do sítio e os objetivos de manejo são diferentes dos preconizados no experimento (DEAN; BALDWIN, 1993). Assim, buscando uma solução alternativa têm-se os diagramas de manejo da densidade, que representam a média do nível de suporte e as relações entre a produção e a densidade populacional, em diferentes estágios do povoamento (NEWTON; WEETMAN, 1994).

O trabalho desenvolvido por Barrio-Anta et al. (2006) formulou um modelo de manejo da densidade em povoamentos de Pinus pinaster através de diagramas. O modelo concebido incluiu informações de volume, biomassa acima do solo, biomassa do povoamento e o estoque de carbono e foi desenvolvido a partir de cinco equações, às quais foram ajustadas de forma simultânea.

A primeira equação relacionou o diâmetro médio com o número de árvores por hectare e altura dominante. Três outras equações relacionaram o volume, a biomassa acima do solo e biomassa do povoamento com o diâmetro médio, o número de árvores e a altura dominante. A última equação relacionou o estoque de carbono com a biomassa acima do solo e a biomassa do povoamento (BARRIO-ANTA et al., 2006).

Em muitos casos de manejo da densidade populacional, o índice de espaçamento relativo é usado para caracterizar o nível de estoque de crescimento. Esse índice foi proposto pela primeira vez para plantações florestais por Hart, em 1928, sendo mais tarde, em 1954, referido como um índice de espaçamento por Becking (1954), sendo calculado pela razão entre a distância média entre árvores e a altura dominante, e expresso em percentagem. Esse índice de espaçamento relativo (RS) é útil no manejo da densidade, pois, do ponto de vista biológico, o crescimento em altura dominante é um dos melhores critérios para o estabelecimento de intervalos de desbaste. A ligação entre o crescimento em altura dominante e produção florestal acrescenta utilidade aos 
diagramas de manejo da densidade para fins de tomada de decisão, por considerar as variações de sítio existentes.

Segundo Newton (1997), os diagramas de manejo da densidade podem ainda ser utilizados para se obter programas de controle populacional para diferentes regimes de manejo, objetivando minimizar a oportunidade temporal, obter de critérios específicos de operacionalidade, controlar o desenvolvimento das árvores durante o estágio inicial de desenvolvimento e otimizar a densidade para os objetivos específicos de qualidade de madeira, vegetação ou de manejo da vida selvagem.

Assim, o presente estudo teve por objetivo elaborar um diagrama de manejo da densidade para povoamentos de Pinus taeda L., para estruturar a produção volumétrica tomando como referência a altura dominante, número de árvores por hectare e índice de espaçamento relativo. O estudo teve por objetivo específico analisar a variação do índice de espaçamento relativo como diâmetro médio de área basal e o número de árvores por hectare e determinar o momento do autodesbaste observando o diâmetro médio de área basal e o número de árvores por hectare.

\section{Material e métodos}

O conjunto de dados utilizado no presente estudo é originado de áreas pertencentes à Empresa Klabin S.A., localizadas no município de Otacílio Costa, Mesorregião Serrana e Microrregião de Campos de Lages. Segundo Alvares et al. (2014), o município está na zona climática do tipo "Cfb", mesotérmico úmido, com verão temperado, precipitação média anual entre 1.600 e $1.900 \mathrm{~mm}$ e temperatura média anual entre 14 e $16^{\circ} \mathrm{C}$. A altitude varia entre $800 \mathrm{a}$ $1.000 \mathrm{~m}$. O solo da área de coleta dos dados é classificado como latossolo bruno, alumínico TB, horizonte A húmico, textura argilosa e relevo suavemente ondulado (EMBRAPA, 1999).

O conjunto de dados foi originado de duas fontes distintas. A primeira fonte de dados foi proveniente de um experimento de espaçamento de 1,5 x 1,0 m e 1,5 x 2,0 m. Em todas as parcelas foram medidas as circunferências a altura do peito e o número de árvores até aos 18 anos. Essas parcelas originaram os dados de número de árvores por hectare e diâmetro médio de área basal correspondentes, tendo originado 50 observações em cada espaçamento, utilizadas para o ajuste do modelo de Tang, definido por:

$$
\ln N(t)=\ln \left(S_{f}\right)-\frac{1}{\gamma} \cdot \ln \left[\left(\frac{d(t)}{d_{o}}\right)^{\beta \cdot \gamma}+\left(\frac{S_{f}}{N_{1}}\right)^{\gamma}-\left(\frac{d_{1}}{d_{o}}\right)^{\beta \cdot \gamma}\right]
$$

Em que: $\mathrm{N}$ = número de árvores por hectare; $\mathrm{t}=$ idade (anos); $\mathrm{S}_{\mathrm{f}}=$ índice de máxima densidade do povoamento; $\gamma$ = índice de autodesbaste; $\mathrm{d}=$ diâmetro médio de área basal $(\mathrm{cm}) ; \mathrm{d}_{\mathrm{o}}=$ diâmetro padrão básico igual a $25 \mathrm{~cm} ; \beta$ = taxa máxima de autodesbaste do povoamento em densidade completa; $\mathrm{N}_{1}=$ primeira observação da densidade do povoamento e $\mathrm{d}_{1}=$ diâmetro médio de área basal inicial $(\mathrm{cm})$.

Na segunda fonte de dados foram tomados dados de um total de 128 parcelas permanentes com dimensão de $20 \times 30 \mathrm{~m}$, instaladas em povoamentos comerciais, com idade variando de 6 a 18 anos, medidas anualmente, manejadas em regime de desbaste por baixo e densidade controlada pelo índice de espaçamento relativo.

O Diagrama de Manejo da Densidade (DMD) desenvolvido incluiu o índice de espaçamento relativo (RS\%) e um sistema de três equações como componentes básicos, descritos por Castedo-Dorado et al. (2009), baseando-se no trabalho pioneiro de Becking (1954), sendo utilizado diferentes formatos para obtenção de um sistema de manejo da densidade, conforme descrito por Jack e Long (1996).

$\mathrm{O}$ índice de espaçamento relativo, utilizado para caracterizar o nível de estoque de crescimento, foi calculado pela razão entre a distância média entre as árvores e altura dominante, expresso em percentagem. Assim, assumindo um espaçamento triangular regular, o índice de 
espaçamento relativo (RS\%) foi determinado por:

$$
R S \%=\frac{\sqrt{20000 /(N \times \sqrt{3})}}{h_{100}} \times 100
$$

Em que: $\mathrm{RS} \%$ = índice de espaçamento relativo (\%); $\mathrm{N}$ = número de árvores (ha) e h100 = altura dominante $(\mathrm{m})$.

Esse índice de espaçamento relativo foi utilizado por ser útil para caracterizar os níveis de crescimento do povoamento, devido a várias razões: é independente da qualidade do sitio e idade, exceto para povoamentos muito jovens; a altura dominante do ponto de vista biológico é o melhor índice para o estabelecimento de intervalos de desbaste; a relação entre o crescimento em altura dominante e produção florestal acrescenta utilidade nestes diagramas para fins de manejo florestal; e é comumente utilizado para controlar a densidade em plantações intensivas.

A construção do diagrama de manejo seguiu os procedimentos descritos abaixo, conforme proposto por Barrio-Anta et al. (2006):

a) Ajuste da equação não linear do diâmetro médio de área basal:

$$
d_{g}=b_{0} \times N^{b_{1}} \times h_{100}^{b_{2}}
$$

b) Ajuste da equação não linear do volume por hectare:

$$
V=b_{3} \times d_{g}^{b_{4}} \times h_{100}^{b_{5}} \times N^{b_{6}}
$$

c) Ajuste do nível de densidade utilizando o índice de espaçamento relativo:

$$
N=\frac{20000 \times 100^{2}}{\sqrt{3} \times R S^{2} \times h_{100}^{2}}
$$

d) Representação das isolinhas do diâmetro médio de área basal usando os parâmetros da equação (3) através da geração constante de dg, entrando com $\mathrm{N}$ através de uma série de h100:

$$
N=\left(\frac{d_{g}}{b_{o} \times h_{100}^{b_{2}}}\right)^{1 / b_{1}}
$$

e) Representação das isolinhas do volume por hectare, introduzindo na equação (4) as equações (3) e (6), respectivamente, obteve-se a solução de $\mathrm{N}$ através de uma série de h100.

$$
N=\left(\frac{V}{b_{3} \times b_{0}^{b_{4}} \times h_{100}^{b_{2} \times b_{4}+b_{5}}}\right)^{1 /\left(b_{1} \times b_{4}+b_{6}\right)}
$$

Em que: $\mathrm{d}_{\mathrm{g}}=$ diâmetro médio de área basal $(\mathrm{cm}) ; \mathrm{N}=$ número de árvores $(\mathrm{ha}) ; \mathrm{h}_{100}=$ altura dominante $(\mathrm{m}) ; \mathrm{V}=$ volume $\left(\mathrm{m}^{3} / \mathrm{ha}\right) ; \mathrm{RS}$ = índice de espaçamento relativo (\%) e $\mathrm{b}_{0}, \mathrm{~b}_{1}, \mathrm{~b}_{2}, \mathrm{~b}_{3}, \mathrm{~b}_{4}, \mathrm{~b}_{5}$ e $\mathrm{b}_{6}=$ parâmetros do modelo.

O ajuste dos modelos de regressão foi realizado através dos procedimentos GLM e NLIN pelo método Gauss-Newton, para regressões lineares e não lineares, respectivamente, no pacote 
Statistical Analysis System (SAS INSTITUTE, 1999). O Diagrama de Manejo da Densidade foi elaborado em planilhas programadas no Microsoft Excel ${ }^{\circledR}$, através da sequência de equações e metodologia anteriormente descritas.

\section{Resultados e discussões}

$\mathrm{Na}$ Tabela 1 encontram-se sumarizados os dados dendrométricos das parcelas utilizadas no presente estudo para elaboração do diagrama de manejo da densidade.

\section{Tabela 1 - Sumarização dos dados amostrados nas parcelas permanentes utilizadas no ajuste dos modelos}

Table 1 - Summarization of the sampled data in the permanent plots used in the adjustment of the models

\begin{tabular}{lcccc}
\hline Variáveis dos povoamentos & Média & Desvio Padrão & Mínimo & Máximo \\
\hline $\mathbf{t}(\mathbf{a n o})$ & 13,7 & 2,1 & 6,0 & 18,0 \\
$\mathbf{N}(\mathbf{h a})$ & 1147 & 38,3 & 304 & 1744 \\
$\mathbf{d}_{\mathbf{g}}(\mathbf{c m})$ & 22,5 & 3,5 & 12,9 & 33,1 \\
$\bar{d}(\mathbf{c m})$ & 22,1 & 3,5 & 12,3 & 32,7 \\
$\bar{h}(\mathbf{m})$ & 17,9 & 3,0 & 10,0 & 27,9 \\
$\mathbf{h}_{\mathbf{1 0 0}}(\mathbf{m})$ & 19,7 & 2,9 & 12,9 & 29,0 \\
$\mathbf{R S}(\mathbf{\%})$ & 15,9 & 2,8 & 12,0 & 23,9 \\
$\mathbf{V}\left(\mathbf{m}^{3} / \mathbf{h a}\right)$ & 324,1 & 102,2 & 56,0 & 573,0 \\
\hline
\end{tabular}

Fonte: Autores (2017)

Em que: $\mathrm{t}$ = idade (ano); $\mathrm{N}=$ número de árvores por hectare; $\mathrm{dg}=$ diâmetro médio de área basal $(\mathrm{cm}) ; \bar{d}=$ diâmetro médio aritmético $(\mathrm{cm}) ; \bar{h}=$ altura média $(\mathrm{m}) ; \mathrm{h}_{100}=$ altura dominante $(\mathrm{m}) ; \mathrm{RS}=$ índice de espaçamento relativo (\%); $\mathrm{V}=$ volume $\left(\mathrm{m}^{3} / \mathrm{ha}\right)$.

Os dados utilizados, originados de parcelas permanentes, de índice de espaçamento relativo (RS) foram relacionados com a altura dominante e o diâmetro médio de área basal, tendo apresentado um coeficiente de determinação igual a 0,61, erro padrão da estimativa igual a 0,0998 e um coeficiente de variação igual a 3,47 \%, sendo expresso pela equação:

$\ln R S=5,16463+0,60208 \times \ln d_{g}-1,37786 \times \ln h_{100}$

Em que: $\ln$ = logaritmo neperiano; RS = índice de espaçamento relativo (\%); dg = diâmetro médio de área basal $(\mathrm{cm}) ; \mathrm{h}_{100}=$ altura dominante $(\mathrm{m})$.

Com a utilização da equação 8 foi obtido um valor de 36,1\% para o Índice de Espaçamento Relativo (RS\%), na idade de 5 anos, com altura dominante de $8,5 \mathrm{~m}$, indicando uma grande disponibilidade de espaço vital. Já o valor do Índice de Espaçamento Relativo de 17,1 \% indica o início da competição dos indivíduos, e foi encontrado aos 20 anos, com altura dominante de 21,9 m. Esses estágios de competitividade já foram demonstrados para Pinus elliottii por Fishwick (1975), que indicou um índice de 16 \% como de elevado nível de competição e acima desse valor como de crescimento livre. 
O ajuste da equação não linear do diâmetro médio de área basal, em função do número de árvores por hectare e altura dominante, apresentou um coeficiente de determinação igual a 0,89, erro padrão da estimativa de 1,104 e um coeficiente de variação igual a $0,78 \%$, sendo expresso pelo modelo:

$$
d_{g}=4,411696 \times N^{-0,10718} \times h_{100}^{0,796261}
$$

Em que: $\mathrm{d}_{\mathrm{g}}=$ diâmetro médio de área basal (cm); $\mathrm{N}$ = número de árvores (ha) e $\mathrm{h}_{100}=$ altura dominante $(\mathrm{m})$.

Da mesma forma, o ajuste da equação não linear do volume em função do número de árvores por hectare e altura dominante apresentou um coeficiente de determinação igual a 0,99, erro padrão da estimativa de 6,654 e um coeficiente de variação igual a 2,05 \%, expresso pelo modelo:

$$
V=0,000019 \times d_{g}^{2,335887} \times h_{100}^{0,764078} \times N^{1,018891}
$$

Em que: $\mathrm{V}=$ volume $\left(\mathrm{m}^{3} / \mathrm{ha}\right) ; \mathrm{dg}=$ diâmetro médio de área basal $(\mathrm{cm}) ; \mathrm{h}_{100}=$ altura dominante $(\mathrm{m})$ e $\mathrm{N}=$ número de árvores (ha).

Foi realizada uma alteração na forma de relacionamento das variáveis de crescimento para inserção do índice de espaçamento relativo. Nesse caso, o diagrama foi construído com o relacionamento do crescimento com índices que representam o tamanho médio da árvore, como por exemplo, volume, altura ou diâmetro, com o número de árvores por hectare. Para isso, diferentes índices de densidade foram utilizados: o índice de densidade de suporte da regra de autodesbaste (TANG et al., 1994), o índice de densidade relativa (DREW; FLEWELLING, 1979) e o índice de espaçamento relativo (YODA et al., 1963), para determinação do estoque volumétrico de madeira.

O Diagrama de Manejo da Densidade (DMD) foi obtido através de uma relação de variáveis dendrométricas, para inclusão do índice de espaçamento relativo para caracterização do estoque de madeira (Figura 1). Ele fornece informações sobre a dimensão do diâmetro médio de áreas basal, o número de árvores por hectare e o volume total, diretamente relacionados com o índice de espaçamento relativo. Esse DMD, assim descrito, permite aos manejadores fazerem comparações rápidas e fáceis do estoque de madeira entre as diferentes opções de regimes desbaste, em que é considerada a produção de madeira.

O diagrama de manejo elaborado permite o cruzamento das variáveis dendrométricas altura dominante e número de árvores por hectare, com variação de 10 a $36 \mathrm{~m}$ e 100 a 3.200 árvores, respectivamente. Foram utilizadas isolinhas com variação de 12 a $28 \%$ para representar o índice de espaçamento relativo (preto), de 12 a $46 \mathrm{~cm}$ para representar o diâmetro médio de área basal (vermelho) e de 100 a $800 \mathrm{~m}^{3}$ para representar o volume por hectare (azul).

Para exemplificar a utilização do diagrama de manejo elaborado, considera-se um povoamento com as variáveis altura dominante e número de árvores por hectare conhecidas de 22 m e 500 árvores, respectivamente. Com o cruzamento de ambas as informações é possível a prognose de: diâmetro médio de área basal igual $26,3 \mathrm{~cm}$; volume por hectare igual a 250 $\mathrm{m}^{3}$ e índice de espaçamento relativo igual a $20,2 \%$, o que indica disponibilidade de espaço de crescimento sobre as árvores. 
Figura 1 - Diagrama de Manejo da Densidade para povoamentos de Pinus taeda L. incluindo as isolinhas de índice de espaçamento relativo

Figure 1 - Density Management Diagram for Pinus taeda L. stands including the relative spacing index isolines

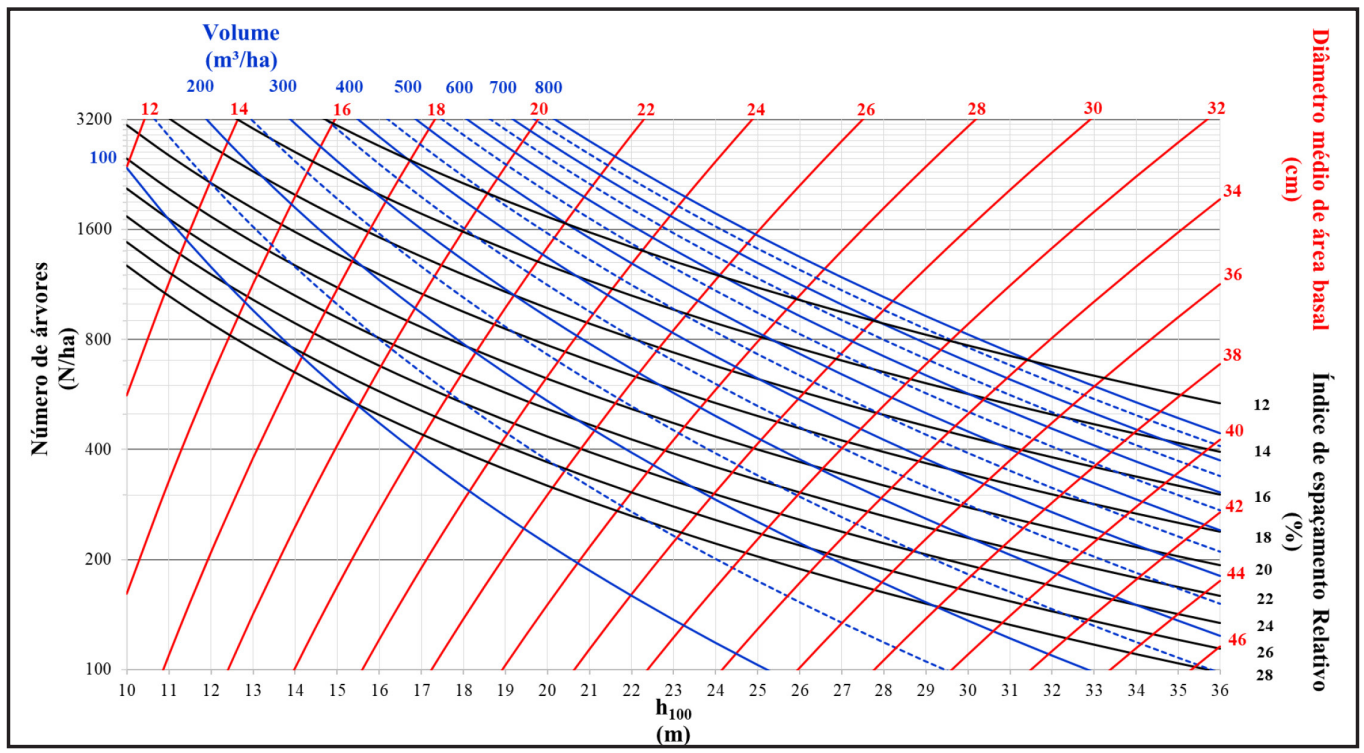

Fonte: Autores (2019)

\section{Conclusões}

O Diagrama de Manejo da Densidade (DMD) desenvolvido permite simular o momento da realização de desbastes em povoamentos de Pinus taeda L., levando em consideração o índice de espaçamento relativo, permitindo obter estimativas do volume por hectare, através da relação com o número de árvores, diâmetro médio de área basal e altura dominante.

\section{Referências}

ALVARES, C. A. et al. Köppen's climate classification map for Brazil. Meteorologische Zeitschrift, Stuttgart, v. 22, n. 6, p. 711-728, 2014.

BARRIO-ANTA, M. et al. An ecoregional model for estimating volume, biomass and carbon pools in maritime pine stands in Galicia (northwestern Spain). Forest Ecology and Management, Amsterdam, v. 223, n. 1/3, p. 24-34, 2006.

BECKING, J. H. Einige Gesichtspunkte für die Durchfürung von vergleichenden Durchfostungsversunchen in Gleichaltrigen Beständen. In: IUFRO Congress, Roma, 1954. Proccedings [...]. Roma: [s. n.], 1954.

CASTEDO-DORADO, F. et al. Development of a stand density management diagram for radiata pine stands including assessment of stand stability. Forestry, Oxford, v. 82, n. 1, p. 1-16, 2009. 
DAVIS, K. P. Forest management: regulation and valuation. 2nd ed. New York: McGraw-Hill, 1966. 519 p.

DEAN, J. T.; BALDWIN, V. C. Using a density-management diagram to develop thinning schedules for loblolly pine plantations. [S. 1.]: USDA Forest Service; Southern Forest Experimental Station, 1993.

DEAN, T. J.; BALDWIN, V. C. Crown management and stand density. In: CARTER, M. (ed.). Growing tress in a greener world: industrial forestry in the 21st Century. Baton Rouge: Louisiana State University Agricultural Center; Louisiana Agricultural Experiment Station, 1996. p. 148-159.

DEL RIO, M. et al. Analysis of diameter-density relationships and self-thinning in non-thinned even-aged Scots pine stands. Forest Ecology and Management, Amsterdam, v. 142, p. 79-87, 2001.

DREW, T. J.; FLEWELLING, J. W. Stand density management: an alternative approach and its application to Douglas-fir plantations. Forest Science, Bethesda, n. 25, p. 518-532, 1979.

EMBRAPA. Sistema brasileiro de classificação de solos. Brasília: EMBRAPA, 1999. 412 p.

FISHWICK, R. W. Uso do percentual de espaçamento relativo de Hart-Becking para o controle dos desbastes. Brasília: PRODEPEF; IBDF, 1975. 7 p.

JACK, S. B.; LONG, J. N. Linkages between silviculture and ecology: analysis of density management diagrams. Forest Ecology and Management, Amsterdam, v. 86, n. 1/3, p. 205-220, 1996.

NEWTON, P. F. Stand density management diagrams: review of their development and utility in stand-level management planning. Forest Ecology and Management, Amsterdam, v. 98, p. 251-265, 1997.

NEWTON, P. F.; WEETMAN, G. F. Stand density management diagram for managed black spruce stands. The Forestry Chronicle, Ottawa. v. 70, n. 1, p. 65-74, 1994.

SAS INSTITUTE. SAS/STAT user's guide. Version 8 (computer manual). Cary, 1999.

SMITH, N. J.; HANN, D. W. A growth model based on the self-thinning rule. Canadian Journal of Forest Research, New Westminster, v. 16, n. 2, p. 330-334, 1986.

TANG, S. et al. A growth and self-thinning model for pure even-aged stands: theory and applications. Forest Ecology and Management, Amsterdam, v. 70, n. 1/3, p. 67-73, 1994.

WESTOBY, M. The place of the self-thinning rule in population dynamics. The American Naturalist, Chicago, v. 118, n. 4, p. 581-587, 1981.

YODA, K. et al. Self-thinning in overcrowed pure stands under cultivated and natural conditions. Journal Biology, [s. 1.], v. 14, p. 107-129, 1963.

ZEIDE, B. Analysis of the 3/2 power law of self-thinning. Forest Science, Bethesda, v. 33, n. 2, p. 17-537, 1987.

ZEIDE, B. Self-thinning and stand density. Forest Science, Bethesda, v. 37, n. 2, p. 517-523, 1991. 